\title{
Simulações com uso de códigos de treliça e decodificação iterativa para o canal aditivo binário
}

\author{
Maria de Lourdes M.G. Alcoforado, Valdemar C. da Rocha Jr. e Garik Markarian
}

\begin{abstract}
Resumo-Este artigo apresenta resultados de simulações computacionais para códigos de treliça sobre o canal aditivo para dois usuários binários, em presença de ruído branco gaussiano aditivo. São comparados os desempenhos de diferentes códigos de treliça com e sem o uso de decodificação iterativa.
\end{abstract}

Palavras-Chave-Acesso múltiplo, canal aditivo, códigos turbo.

Abstract-This paper presents simulation results for trellis codes over the two-user binary adder channel, in the presence of additive white Gaussian noise. The performance of different trellis codes is compared with and without iterative decoding.

Keywords-Multiple access, additive channel, turbo codes.

\section{INTRODUÇÃO}

Este artigo utiliza a decodificação iterativa [1], [2] em sistemas de comunicações que empregam canal de acesso múltiplo, isto é, um meio de transmissão no qual mais de um usuário pode acessá-lo simultaneamente, com a saída do canal sendo uma combinação dos sinais enviados pelos usuários ativos. Em particular, é dada ênfase ao caso em que dois usuários binários podem transmitir simultaneamente em um canal aditivo para um único receptor. Este canal é chamado de canal aditivo com dois usuários binários (2-BAC)[3]-[5].

São apresentadas simulações computacionais de sistemas codificados, em presença de ruído branco gaussiano aditivo, e utilizadas condições de decodibilidade única para códigos de treliça adaptados ao canal 2-BAC [6]-[7].

$\mathrm{Na}$ Seção II é descrito o conceito de treliça para dois usuários. Na Seção III descreve-se um esquema de codificação para obtenção de códigos unicamente decodificáveis para o 2BAC. Este esquema consiste na concatenação em série de um par de códigos de bloco unicamente decodificáveis no 2-BAC com um par de códigos convolucionais sistemáticos [7], [8]. Na Seção IV são apresentados os resultados das simulações, nas quais os codificadores convolucionais utilizados pelos dois usuários podem ser idênticos ou distintos. Na $S e c ̧ a ̃ o ~ V$ são apresentadas as conclusões.

\section{TRELIÇA PARA O 2-BAC}

Nesta seção está descrita a construção de Peterson e Costello [9] de uma treliça para o 2-BAC, denominada treliça para dois usuários, a partir das treliças individuais de cada usuário.

Maria de Lourdes M.G. Alcoforado, Escola Politécnica de Pernambuco, Departamento de Engenharia Elétrica, 50750-470, Recife, PE, Brasil, Email: mlmga@upe.poli.br. Valdemar C. da Rocha Jr., Grupo de Pesquisa em Comunicações - CODEC, Departamento de Eletrônica e Sistemas, Universidade Federal de Pernambuco, Caixa Postal 7800, 50711-970, Recife, PE, Brasil, Email: vcr@ufpe.br. Garik Markarian, Institute of Integrated Information Systems, University of Leeds, LS2 9JT, England, Email: G.Markarian@leeds.ac.uk.

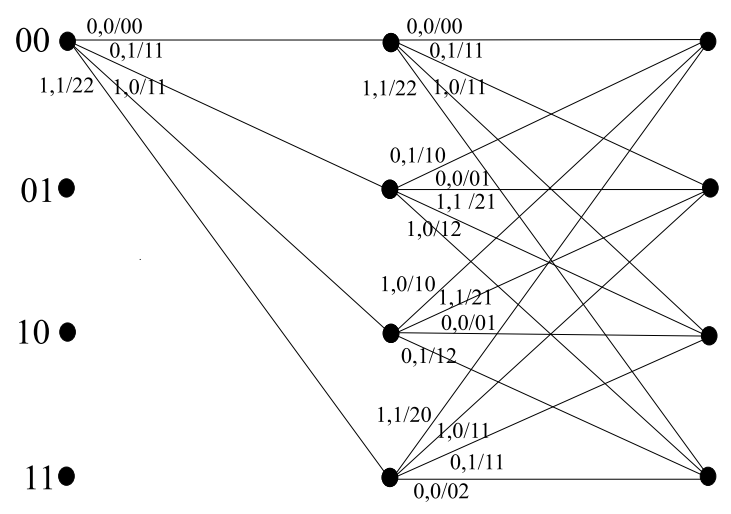

Fig. 1. Treliça para dois usuários binários em que, para cada usuário, é usado um mesmo código convolucional com matriz geradora $G(D)=$ $\left[\begin{array}{ll}1 & \frac{1}{1+D}\end{array}\right]$. Os rótulos nos ramos $\left(u_{k}, d_{k} / x_{1} x_{2}\right)$ correspondem, respectivamente, ao par de símbolos de informação $u_{k}, d_{k}$ dos usuários 1 e 2 e à saída $x_{1} x_{2}$ do 2-BAC sem ruído.

Deve-se supor que as treliças de cada usuário são iniciadas num mesmo instante de tempo e que são considerados pares de ramos (um ramo de cada treliça) que ocorrem em um mesmo intervalo de tempo. Ao estado $S_{k}=s_{i}$, na treliça do usuário 1 , e ao estado $S_{k}^{\prime}=s_{r}$, na treliça do usuário 2, associa-se na treliça para dois usuários o estado denotado por $s_{i} s_{r}$. Cada par de ramos, ocorrendo em um mesmo intervalo de tempo, nas respectivas treliças de cada um dos dois usuários, é associado a um único ramo na treliça para dois usuários. Dito de outra forma, se num dado intervalo de tempo o ramo correspondente ao usuário 1 segue do estado $s_{i}$ para o estado $s_{j}$, e o ramo correspondente ao usuário 2 segue do estado $s_{r}$ para o estado $s_{l}$, então na treliça para dois usuários corresponde um único ramo seguindo do estado $s_{i} s_{r}$ para o estado $s_{j} s_{l}$. Se a treliça de cada usuário tem, respectivamente, $L_{1}$ e $L_{2}$ estados, a treliça para dois usuários terá $L_{1} L_{2}$ estados. $\mathrm{O}$ conceito de treliça para dois usuários é ilustrado por meio de um exemplo.

Exemplo 2.1: Supõe-se uma situação hipotética, apenas para ilustrar a construção da treliça para dois usuários, na qual um mesmo código convolucional é usado por cada usuário do 2-BAC. Seja $\mathcal{C}$ o código convolucional recursivo sistemático com taxa assintótica $1 / 2$ e matriz geradora polinomial:

$$
G(D)=\left[\begin{array}{ll}
1 & \frac{1}{1+D}
\end{array}\right]
$$

Como o código tem apenas um elemento de memória, a treliça de cada usuário possui dois estados, i.e., $L_{1}=L_{2}=2$, e a treliça para dois usuários terá $L_{1} L_{2}=4$ estados como ilustrado na Figura 1. 


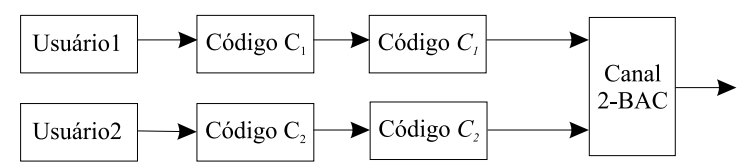

Fig. 2. Modelo de construção de código unicamente decodificável para o 2-BAC

\section{DeCodificaÇÃo Iterativa PARA o CANAL Aditivo COM DOIS USUÁRIOS BINÁRIOS USANDO Códigos DE TRELIÇA}

Nesta secção é mostrado um método de construção de códigos de treliça, a partir da concatenação em série de um par de códigos unicamente decodificáveis para o 2-BAC com um par de códigos convolucionais [7], [8].

Considere um par de códigos convolucionais $\left(\mathcal{C}_{1}, \mathcal{C}_{2}\right)$ recursivos e sistemáticos com taxas assintóticas iguais a $\mathrm{k} / n$, com memórias iguais a $m_{1}$ e $m_{2}$, respectivamente [10, p.303308]. Seja $\left(\mathbf{C}_{1}, \mathbf{C}_{2}\right)$ um par de códigos de bloco unicamente decodificáveis no 2-BAC.

$\mathrm{O}$ código para o usuário 1 é construído a partir de $\mathcal{C}_{1} \mathrm{e}$ de $\mathbf{C}_{1}$ do seguinte modo. $\mathrm{O}$ usuário 1 envia suas mensagens para o codificador de $\mathbf{C}_{1}$, e as palavras-código resultantes de $\mathbf{C}_{1}$ são enviadas como mensagens para o codificador de $\mathcal{C}_{1}$. Desta forma o usuário 1 estará se servindo de um dicionário contendo um subconjunto das palavras-código de $\mathcal{C}_{1}$, escolhidas de acordo com as "mensagens" alimentadas por $\mathbf{C}_{1}$ ao codificador de $\mathcal{C}_{1}$. A codificação para o usuário 2 é semelhante, empregando o código $\mathcal{C}_{2}$ e o código $\mathbf{C}_{2}$. Conseqüentemente, o usuário 2 estará se servindo de um dicionário contendo um subconjunto das palavras-código de $\mathcal{C}_{2}$, escolhidas de acordo com as "mensagens" alimentadas por $\mathbf{C}_{2}$ ao codificador de $\mathcal{C}_{2}$.

Essencialmente a operação de codificação desempenhada por cada usuário é uma concatenação em série dos seus respectivos códigos de bloco com o código convolucional, conforme ilustrado na Figura 2.

Como $\mathcal{C}_{1}$ e $\mathcal{C}_{2}$ são sistemáticos e possuem taxas assintóticas iguais, a soma aritmética bit a bit das palavras-código de $\mathcal{C}_{1}$ produzidas pelo usuário 1 e das palavras-código de $\mathcal{C}_{2}$ produzidas pelo usuário 2, é unicamente decodificável. Esta afirmação procede porque o par $\left(\mathbf{C}_{1}, \mathbf{C}_{2}\right)$ é unicamente decodificável no 2-BAC e a soma aritmética das palavras-código de $\mathbf{C}_{1}$ e $\mathbf{C}_{2}$ aparecem na seção de informação da soma aritmética das palavras-código de $\mathcal{C}_{1}$ e $\mathcal{C}_{2}$.

$\mathrm{O}$ uso dos códigos $\mathbf{C}_{1}$ e $\mathbf{C}_{2}$ leva à eliminação de alguns ramos e, algumas vezes, leva à eliminação de alguns estados na treliça para dois usuários. Em outras palavras, serão eliminados caminhos nas treliças dos códigos convolucionais empregados, evitando assim problemas de ambigüidade na decodificação.

Se a taxa do par $\left(\mathbf{C}_{1}, \mathbf{C}_{2}\right)$ é $R$, segue desta construção que $R_{C}=\left(\frac{k}{n}\right) R$ é a taxa do código construído para o 2-BAC. Portanto, se $\mathcal{C}_{1}$ e $\mathcal{C}_{2}$ forem escolhidos para serem códigos com taxas assintóticas aproximadamente iguais a $1, R_{C}$ terá um valor muito próximo a $R$. Isto significa que se $R$ alcançar o valor máximo da taxa de trasmissão (capacidade) para o 2-BAC, então $R_{C}$ também alcançará este valor.

\section{A. O Codificador}

Considere o modelo de construção de código unicamente decodificável para o 2-BAC ilustrado na Figura 2. Considere que, a partir de agora, o codificador para $\mathcal{C}_{1}$, utiliza o esquema de concatenação paralela introduzido em [1] e [2]. Desta forma, o codificador para $\mathcal{C}_{1}$ é formado pela concatenação paralela de dois códigos convolucionais recursivos componentes, $\mathcal{C}_{1}^{-}$e $\mathcal{C}_{1}^{\mid}$, não necessariamente iguais. As entradas de ambos os codificadores componentes utilizam os mesmos bits de informação $u_{k}$, mas em uma ordem diferente, devido à presença do entrelaçador. Similarmente, o codificador para $\mathcal{C}_{2}$ é formado pela concatenação paralela de dois códigos convolucionais recursivos componentes, $\mathcal{C}_{2}^{-}$e $\mathcal{C}_{2}^{\mid}$, não necessariamente iguais. As entradas de ambos os codificadores componentes utilizam os mesmos bits de informação $d_{k}$, mas em uma ordem diferente, devido à presença do entrelaçador, que deve ser idêntico ao entrelaçador utilizado para $\mathcal{C}_{1}$. A taxa de transmissão de $\mathcal{C}_{1}$ deve ser igual à taxa de transmissão de $\mathcal{C}_{2}$.

Considere, sem perda de generalidade, que cada codificador recursivo sistemático tem taxa de transmissão assintótica $1 / n$ e $M$ estados, para ambos usuários. Tem-se que as sequiências de símbolos de informação para o usuário 1 e usuário 2 são representadas respectivamente por:

$$
\begin{gathered}
\boldsymbol{u}=\boldsymbol{u}_{1}^{N}=\left\{u_{1}, u_{2}, \ldots, u_{k}, \ldots, u_{N}\right\}, \\
\boldsymbol{d}=\boldsymbol{d}_{1}^{N}=\left\{d_{1}, d_{2}, \ldots, d_{k}, \ldots, d_{N}\right\} .
\end{gathered}
$$

As seqüências código associadas ao usuário 1 e ao usuário 2 são representadas respectivamente por:

$$
\begin{gathered}
\boldsymbol{v}=\boldsymbol{v}_{1}^{N}=\left\{\boldsymbol{v}_{1}, \boldsymbol{v}_{2}, \ldots, \boldsymbol{v}_{k}, \ldots, \boldsymbol{v}_{N}\right\} \\
\boldsymbol{w}=\boldsymbol{w}_{1}^{N}=\left\{\boldsymbol{w}_{1}, \boldsymbol{w}_{2}, \ldots, \boldsymbol{w}_{k}, \ldots, \boldsymbol{w}_{N}\right\} .
\end{gathered}
$$

em que $\boldsymbol{v}_{k}=\left(v_{k}^{(0)}, v_{k}^{(1)}, \ldots, v_{k}^{(n-1)}\right)=\left(u_{k}, v_{k}^{(1)}, \ldots, v_{k}^{(n-1)}\right)$, é a saída associada a cada símbolo de informação do usuário 1 e similarmente, $\boldsymbol{w}_{k}=\left(w_{k}^{(0)}, w_{k}^{(1)}, \ldots, w_{k}^{(n-1)}\right)=$ $\left(d_{k}, w_{k}^{(1)}, \ldots, w_{k}^{(n-1)}\right)$, é a saída associada a cada símbolo de informação do usuário 2. $v_{k}^{(0)}$ e $w_{k}^{(0)}$ são as saídas sistemáticas dos codificadores para o usuário 1 e para o usuário 2 , respectivamente.

Na Figura 3 está ilustrado o codificador para $\mathcal{C}_{1}$, para códigos convolucionais constituintes com taxa $1 / 2$. o codificador resultante tem taxa $1 / 3$.

\section{B. O canal 2-BAC}

Os termos $\boldsymbol{v}_{1}^{N}$ e $\boldsymbol{w}_{1}^{N}$ são as entradas para um canal aditivo ruidoso sem memória, com dois usuários binários. $\mathrm{O}$ ruído aqui considerado é o ruído branco gaussiano aditivo. A sequiência de sub-blocos na treliça para dois usuários é dada por

$$
\boldsymbol{x}=\boldsymbol{x}_{1}^{N}=\left\{\boldsymbol{x}_{1}, \boldsymbol{x}_{2}, \ldots, \boldsymbol{x}_{k}, \ldots, \boldsymbol{x}_{N}\right\},
$$

em que $\boldsymbol{x}_{k}=\left(x_{k}^{(0)}, x_{k}^{(1)}, \ldots, x_{k}^{(n-1)}\right)$. A variável aleatória $x_{k}^{(j)}, j=0, \ldots, n-1$, no instante de tempo $k$, é definida por meio da seguinte igualdade

$$
x_{k}^{(j)}=\left(2 v_{k}^{(j)}-1\right)+\left(2 w_{k}^{(j)}-1\right), j=0, \ldots, n-1 .(2)
$$




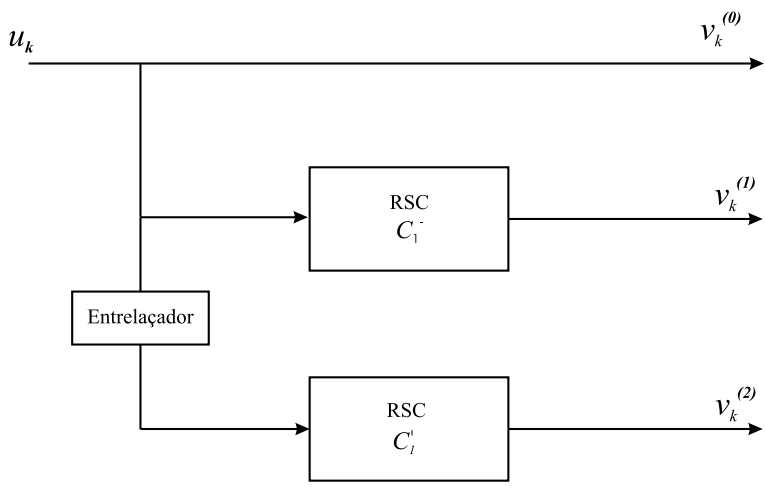

Fig. 3. Esquema de concatenação paralela para o codificador de $\mathcal{C}_{1}$.

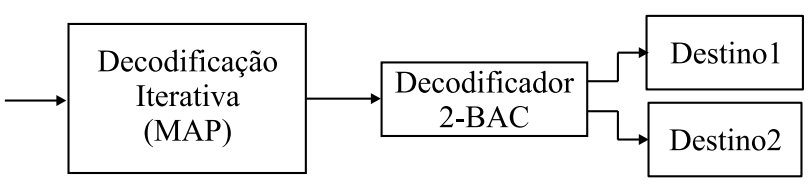

Fig. 4. O decodificador empregado utiliza a decodificação iterativa para detectar a seqüência ternária mais provável e em seguida usa o decodificador 2-BAC para separar a informação relativa aos usuários 1 e 2.

A saída do canal é a seqüência recebida

$$
\boldsymbol{r}=\boldsymbol{r}_{1}^{N}=\left\{\boldsymbol{r}_{1}, \boldsymbol{r}_{2}, \ldots, \boldsymbol{r}_{k}, \ldots, \boldsymbol{r}_{N}\right\}
$$

em que $\boldsymbol{r}_{k}=\left(r_{k}^{(0)}, r_{k}^{(1)}, \ldots, r_{k}^{(n-1)}\right)$. A variável aleatória $r_{k}^{(j)}, j=0, \ldots, n-1$, no instante de tempo $k$, é definida pela seguinte igualdade

$$
r_{k}^{(j)}=x_{k}^{(j)}+q_{k}^{(j)}, j=0, \ldots, n-1,
$$

em que $q_{k}^{(j)}$ são amostras independentes de ruído, todas com a mesma variância $\sigma^{2}$ e média zero.

\section{O Decodificador}

O decodificador em questão (Figura 4), utiliza a decodificação iterativa [11] para detectar a seqüência ternária mais provável e em seguida usa o decodificador 2-BAC para separar a informação relativa aos usuários 1 e 2, utilizando para isto o par $\left(\mathbf{C}_{1}, \mathbf{C}_{2}\right)$ de códigos de bloco unicamente decodificáveis para o 2-BAC.

O algoritmo aqui utilizado é o BCJR [8], [12] que faz uso da treliça para dois usuários definida na Seção II e calcula as razões de log-verossimilhança $\Lambda_{1}\left(u_{k}, d_{k}\right), \Lambda_{2}\left(u_{k}, d_{k}\right)$ e $\Lambda_{3}\left(u_{k}, d_{k}\right)$ associadas ao par dos símbolos de informação $\left(u_{k}, d_{k}\right)$, relativos aos usuários 1 e 2 , repectivamente.

$$
\begin{aligned}
& \Lambda_{1}\left(u_{k}, d_{k}\right)=\log \frac{\mathrm{P}\left\{u_{k}=1, d_{k}=0 \mid \boldsymbol{r}\right\}}{\mathrm{P}\left\{u_{k}=0, d_{k}=0 \mid \boldsymbol{r}\right\}}, \\
& \Lambda_{2}\left(u_{k}, d_{k}\right)=\log \frac{\mathrm{P}\left\{u_{k}=1, d_{k}=1 \mid \boldsymbol{r}\right\}}{\mathrm{P}\left\{u_{k}=0, d_{k}=0 \mid \boldsymbol{r}\right\}}, \\
& \Lambda_{3}\left(u_{k}, d_{k}\right)=\log \frac{\mathrm{P}\left\{u_{k}=0, d_{k}=1 \mid \boldsymbol{r}\right\}}{\mathrm{P}\left\{u_{k}=0, d_{k}=0 \mid \boldsymbol{r}\right\}},
\end{aligned}
$$

em que $\mathrm{P}\left\{u_{k}=i, d_{k}=s \mid \boldsymbol{r}\right\}, i=0,1, s=0,1$, é a probabilidade a posteriori do par $\left(u_{k}, d_{k}\right)$ de símbolos de informação.

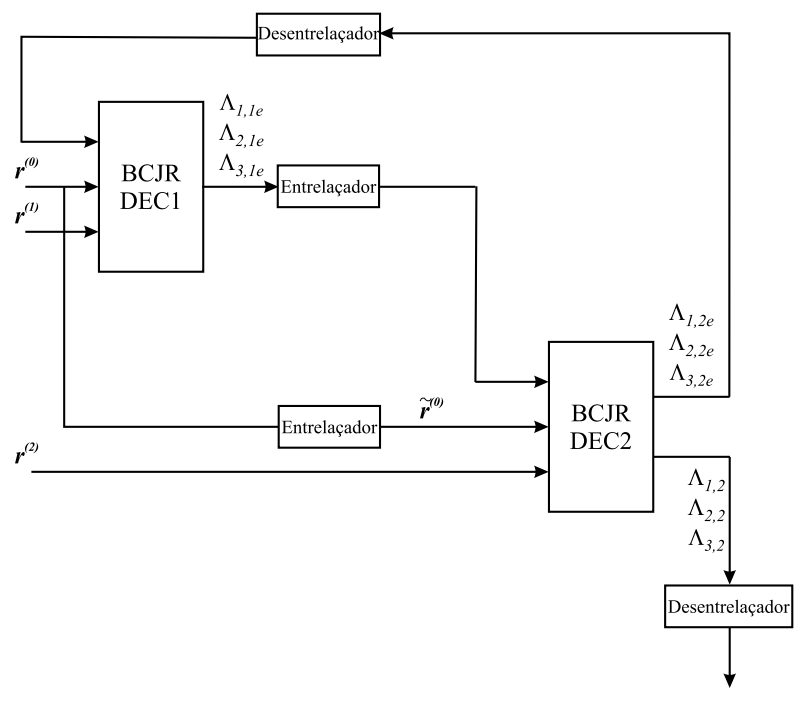

Fig. 5. O decodificador utiliza o princípio da decodificação iterativa e consiste de dois decodificadores componentes concatenados em série. É usado para detectar a seqüência ternária mais provável.

Verificando a Figura 4 e em especial o bloco relacionado à decodificação iterativa (MAP), suponha que a partir de agora ele consiste de dois decodificadores componentes concatenados em série, como pode ser visto na Figura 5.

$\mathrm{Na}$ entrada do primeiro decodificador BCJR, denotado por DEC1, tem-se as sequiências recebidas $\boldsymbol{r}^{(0)}=$ $\left\{r_{1}^{(0)}, r_{2}^{(0)}, \ldots, r_{N}^{(0)}\right\}$ e $\boldsymbol{r}^{(1)}=\left\{r_{1}^{(1)}, r_{2}^{(1)}, \ldots, r_{N}^{(1)}\right\}$, em que $r_{k}^{(j)}$ foi definido em (3). DEC1 então produz as saídas suaves $\left(\Lambda_{1,1}\left(u_{k}, d_{k}\right), \Lambda_{2,1}\left(u_{k}, d_{k}\right), \Lambda_{3,1}\left(u_{k}, d_{k}\right)\right)$, que são entrelaçadas e usadas para produzir estimativas das probabilidades a priori dos pares de seqüências de informação para o segundo decodificador BCJR, denotado por DEC2. A notação $\Lambda_{1,1}\left(u_{k}, d_{k}\right), \Lambda_{2,1}\left(u_{k}, d_{k}\right), \Lambda_{3,1}\left(u_{k}, d_{k}\right)$ é usada para denotar as saídas suaves $\Lambda_{1}\left(u_{k}, d_{k}\right), \Lambda_{2}\left(u_{k}, d_{k}\right)$ e $\Lambda_{3}\left(u_{k}, d_{k}\right)$ respectivamente, associadas com DEC1.

$\mathrm{Na}$ entrada de DEC2 estão as seqüências recebidas $\tilde{\boldsymbol{r}}^{(0)}$ e $\boldsymbol{r}^{(2)}=\left\{r_{1}^{(2)}, r_{2}^{(2)}, \ldots, r_{N}^{(2)}\right\}$. A seqüência $\tilde{\boldsymbol{r}}^{(0)}$ corresponde à seqüência $\boldsymbol{r}^{(0)}$ entrelaçada. DEC2 também produz saídas suaves $\left(\Lambda_{1,2}\left(u_{k}, d_{k}\right), \Lambda_{2,2}\left(u_{k}, d_{k}\right), \Lambda_{3,2}\left(u_{k}, d_{k}\right)\right)$, cuja notação é usada para indicar que as saídas suaves $\Lambda_{1}\left(u_{k}, d_{k}\right), \Lambda_{2}\left(u_{k}, d_{k}\right)$ e $\Lambda_{3}\left(u_{k}, d_{k}\right)$ estão associadas com DEC2. Estas saídas suaves são usadas para melhorar a estimativa das probabilidades a priori dos pares de seqüências $\left(u_{k}, d_{k}\right)$ de informação na entrada de DEC1.

DEC2 estima as razões de log-verossimilhança $\Lambda_{1,2}\left(u_{k}, d_{k}\right), \Lambda_{2,2}\left(u_{k}, d_{k}\right)$ e $\Lambda_{3,2}\left(u_{k}, d_{k}\right)$.

$\Lambda_{1,2 e}\left(u_{k}, d_{k}\right), \quad \Lambda_{2,2 e}\left(u_{k}, d_{k}\right)$ e $\Lambda_{3,2 e}\left(u_{k}, d_{k}\right)$ são as informações extrínsicas para DEC2, que dependem das informações redundantes supridas pelos codificadores $\mathcal{C}_{1}^{\mid}$e $\mathcal{C}_{2}^{\mid}$. As informações extrínsicas de DEC2 são usadas como estimativas das probabilidades a priori para DEC1. $\hat{\Lambda}_{1,2 e}\left(u_{k}, d_{k}\right)$, $\hat{\Lambda}_{2,2 e}\left(u_{k}, d_{k}\right)$ e $\hat{\Lambda}_{3,2 e}\left(u_{k}, d_{k}\right)$ correspondem, respectivamente, aos valores $\Lambda_{1,2 e}\left(u_{k}, d_{k}\right), \Lambda_{2,2 e}\left(u_{k}, d_{k}\right)$ e $\Lambda_{3,2 e}\left(0000 u_{k}, d_{k}\right)$ desentrelaçados. 


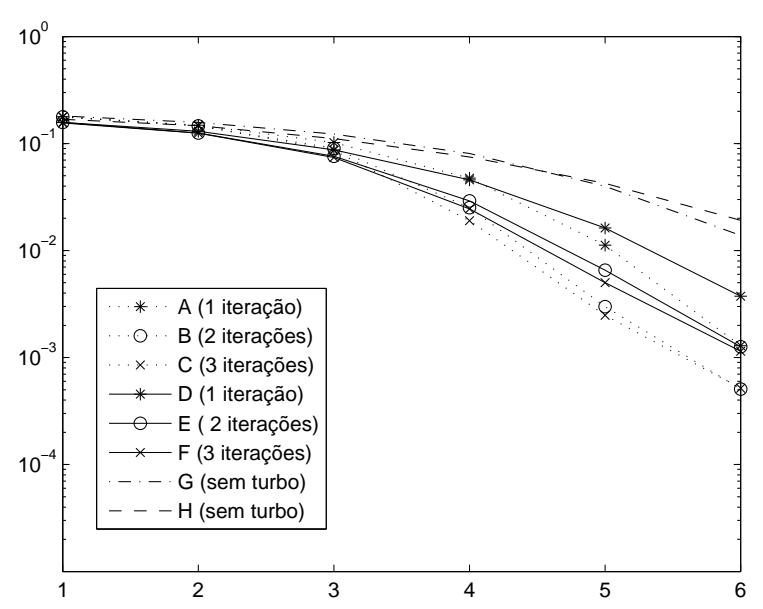

Fig. 6. Curvas relacionadas ao usuário 1. Os casos para os quais $\mathcal{C}_{1}^{-}=\mathcal{C}_{1}^{\mid}=$ $\mathcal{C}_{2}^{-}=\mathcal{C}_{2}^{\mid}$têm matrizes geradoras polinomiais $G(D)=\left[\begin{array}{cc}1 & \frac{1+D^{2}}{1+D+D^{2}}\end{array}\right]$ estão ilustrados em: A, B, C e G; Os casos para os quais $\mathcal{C}_{1}^{-}=\mathcal{C}_{1}^{\mid}=\mathcal{C}_{2}^{-}=\mathcal{C}_{2}^{\mid}$ têm matrizes geradoras polinomiais $G(D)=\left[\begin{array}{ll}1 & \frac{D+D^{2}}{1+D+D^{2}}\end{array}\right]$ estão ilustrados em: D, E, F e H.

\section{RESUltados DAS SIMULAÇÕES}

Esta seção tem como objetivo apresentar resultados de simulações computacionais quando o esquema de concatenação ilustrado na Figura 2 é usado e também comparar as curvas relacionando a probabilidade de erro por bit versus relação sinal ruído para dois usuários, para o caso em que é usado apenas o algoritmo BCJR, e para o caso em que é utilizada a decodificação iterativa. Todas as implemetações foram feitas através de simulações com a utilização da ferramenta MATLAB 6.5 [13]. É assumida a presença de ruído branco gaussiano aditivo. Em todas as curvas apresentadas, os códigos de bloco utilizados para os usuários 1 e 2 são, respectivamente, $C_{1}=\{01,10\}$ e $C_{2}=\{00,01,11\}$. Para a decodificação iterativa é usado o entrelaçador de BerrouGlaviex[1], [2] com comprlimento 512. Em todos os casos investigados, como os códigos de bloco utilizados são sempre os mesmos, é comparado o efeito de alterar os códigos convolucionais (Figuras 6 a 13), isto é, compara-se o efeito de variar os codificadores convolucionais para $\mathcal{C}_{1}^{-}=\mathcal{C}_{1}^{\mid}$e $\mathcal{C}_{2}^{-}=\mathcal{C}_{2}^{\mid}$, respectivamente. É importante observar que nos casos das Figuras 6 a 11, os codificadores convolucionais dos dois usuários são iguais, isto é, $\mathcal{C}_{1}^{-}=\mathcal{C}_{1}^{\mid}=\mathcal{C}_{2}^{-}=\mathcal{C}_{2}^{\mid}$. Em relação as Figuras 12 e13, os codificadores convolucionais dos dois usuários são diferentes, isto é $\mathcal{C}_{1}^{-}=\mathcal{C}_{1}^{\mid}$diferentes de $\mathcal{C}_{2}^{-}=\mathcal{C}_{2}^{\mid}$. Em todos os casos apresentados (Figuras 6 a 13) verifica-se a vantagem do uso da decodificação iterativa em relação ao caso em que é usado apenas o algoritmo BCJR.

\section{Conclusões}

Foram usados códigos convolucionais com pequeno comprimento de restrição a fim de usufruir de uma menor complexidade computacional. Verifica-se que em praticamente todos os casos apresentados, três iterações são suficientes para

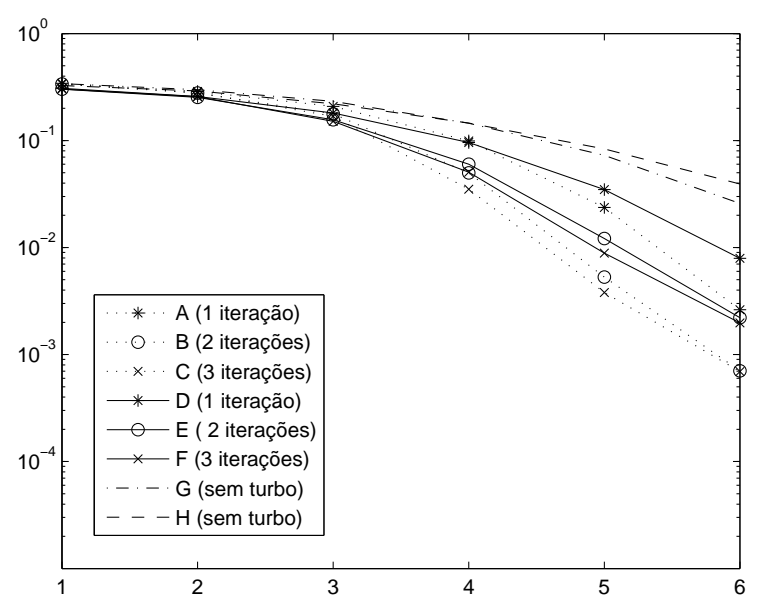

Fig. 7. Curvas relacionadas ao usuário 2. Os casos para os quais $\mathcal{C}_{1}^{-}=\mathcal{C}_{1}^{\mid}=$ $\mathcal{C}_{2}^{-}=\mathcal{C}_{2}^{\mid}$têm matrizes geradoras polinomiais $G(D)=\left[\begin{array}{cc}1 & \frac{1+D^{2}}{1+D+D^{2}}\end{array}\right]$ estão ilustrados em: A, B, C e G; Os casos para os quais $\mathcal{C}_{1}^{-}=\mathcal{C}_{1}^{\mid}=\mathcal{C}_{2}^{-}=\mathcal{C}_{2}^{\mid}$ têm matrizes geradoras polinomiais $G(D)=\left[\begin{array}{ll}1 & \frac{D+D^{2}}{1+D+D^{2}}\end{array}\right]$ estão ilustrados em: D, E, F e H.

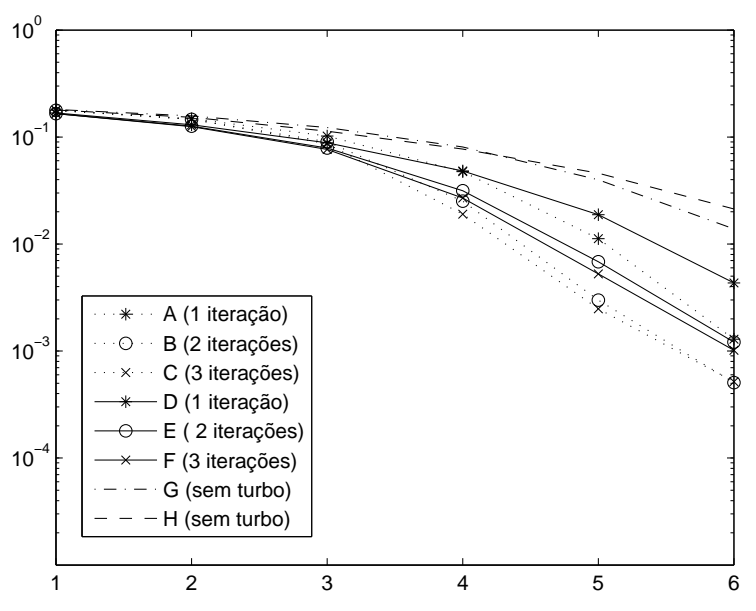

Fig. 8. Curvas relacionadas ao usuário 1. Os casos para os quais $\mathcal{C}_{1}^{-}=\mathcal{C}_{1}^{\mid}=$ $\mathcal{C}_{2}^{-}=\mathcal{C}_{2}^{\mid}$têm matrizes geradoras polinomiais $G(D)=\left[\begin{array}{ll}1 & \frac{1+D^{2}}{1+D+D^{2}}\end{array}\right]$ estão ilustrados em: A, B, C e G; Os casos para os quais $\mathcal{C}_{1}^{-}=\mathcal{C}_{1}^{\mid}=\mathcal{C}_{2}^{-}=\mathcal{C}_{2}^{\mid}$ têm matrizes geradoras polinomiais $G(D)=\left[\begin{array}{ll}1 & \frac{D}{1+D+D^{2}}\end{array}\right]$ estão ilustrados em: D, E, F e H.

conseguir a menor probabilidade de erro deste esquema. $\mathrm{O}$ caso em que os codificadores convolucionais do usuário 1 são diferentes dos codificadores convolucionais do usuário 2, também ilustram a vantagem de utilização de decodificação iterativa com uso do esquema da Figura 2.

\section{AgRAdECIMENTOS}

Valdemar C. da Rocha Jr. agradece ao Conselho Nacional de Desenvolvimento Científico e Tecnológico - CNPq, pelo apoio recebido através do Projeto 305226/2003-7. 


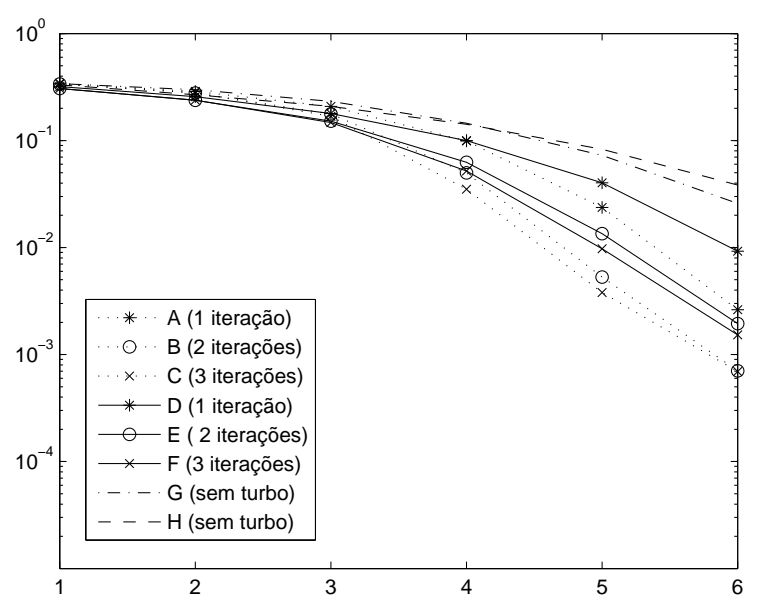

Fig. 9. Curvas relacionadas ao usuário 2. Os casos para os quais $\mathcal{C}_{1}^{-}=\mathcal{C}_{1}^{\mid}=$ $\mathcal{C}_{2}^{-}=\mathcal{C}_{2}^{\mid}$têm matrizes geradoras polinomiais $G(D)=\left[\begin{array}{cc}1 & \frac{1+D^{2}}{1+D+D^{2}}\end{array}\right]$ estão ilustrados em: A, B, C e G; Os casos para os quais $\mathcal{C}_{1}^{-}=\mathcal{C}_{1}^{\mid}=\mathcal{C}_{2}^{-}=\mathcal{C}_{2}^{\mid}$ têm matrizes geradoras polinomiais $G(D)=\left[\begin{array}{ll}1 & \frac{D}{1+D+D^{2}}\end{array}\right]$ estão ilustrados em: D, E, F e H.

\section{REFERÊNCIAS}

[1] C. Berrou, A. Glavieux and P. Thitimajshima, "Near Shannon Limit, Error-Correcting Coding and Decoding: Turbo Codes ", IEEE International Conference on Communications (ICC'93), vol. 2/3, pp.10641071,May 1993

[2] C. Berrou and Alain Glavieux, "Near Optimum Error Correcting Coding And Decoding: Turbo-Codes", IEEE Trans. Commun.,vol. 44, no.10, pp.1261-1271, October 1996.

[3] C. E. Shannon, "Two-way communication channels", in Proc. 4th Berkeley Symp. Math. Stat. Prob., vol. 1, pp.611-644, 1961. Reprinted in Key Papers in the Development of Information Theory., D. Slepian, Ed. New York, IEEE Press, pp.339-372, 1974.

[4] H. A. Cabral Codificação para Canal de Acesso Múltiplo Síncrono. Recife, Brasil, 1994. Dissertação (Mestrado em Engenharia Elétrica)Departamento de Eletrônica e Sistemas, UFPE.

[5] M. L. M. G. Alcoforado, Implementação Algorítmica de Códigos Lineares para o Canal Aditivo com Dois Usuários Binários. Recife, Brasil, 1999. Dissertação (Mestrado em Engenharia Elétrica)- Departamento de Eletrônica e Sistemas, UFPE.

[6] V. C. da Rocha Jr. and M.L.M.G. Alcoforado, "Trellis code construction for the 2-user binary adder channel", in the Proceedings of $11^{\text {th }}$ International Conference on Telecommunications, 1-5 August 2004, Fortaleza, Ceará, Brazil, Editors J. Neuman and P. Dini, Springer Verlag.

[7] V. C. da Rocha Jr. and M.L.M.G. Alcoforado, "Uniquely decodable trellis codes for the two user binary adder channel", International Symposium on Information Theory and its Applications, 10-13 October 2004, Parma, Italy.

[8] M. L. M. G. Alcoforado, V. C. da Rocha Jr. and G. Markarian "Turbo Convolutional Codes for the Binary Adder Channel" VIII International Symposium on Communication Theory and its Application, July Ambleside, UK.2005.

[9] R. Peterson and D. J. Costello, Jr., "Binary convolutional codes for a multiple-access channel", IEEE Trans. on Info. Theory, v. 25, Number 1, p.101-105, January 1979.

[10] S. Lin and D. Costello Jr., Error Control Coding: Fundamentals and Applications, Prentice-Hall Inc., Englewood Cliffs, New Jersey, USA, 1983.

[11] J. Hagenauer, "Iterative Decoding of Binary Block and Convolutional Codes", IEEE Trans. on Inform. Theory, vol. 42, no.2, pp.429-445, March 1996.

[12] L. R. Bahl, J. Cocke, F. Jelinek and J. Raviv, "Optimal decoding of linear codes for minimizing symbol error rate", IEEE Trans. Inform. Theory, vol. IT-20, pp.284-287, March 1974.

[13] HANSELMAN, D.; LITTLEFIELD, B. Matlab, Guia do Usuário. São Paulo, Brasil: Makron Books, 1999.

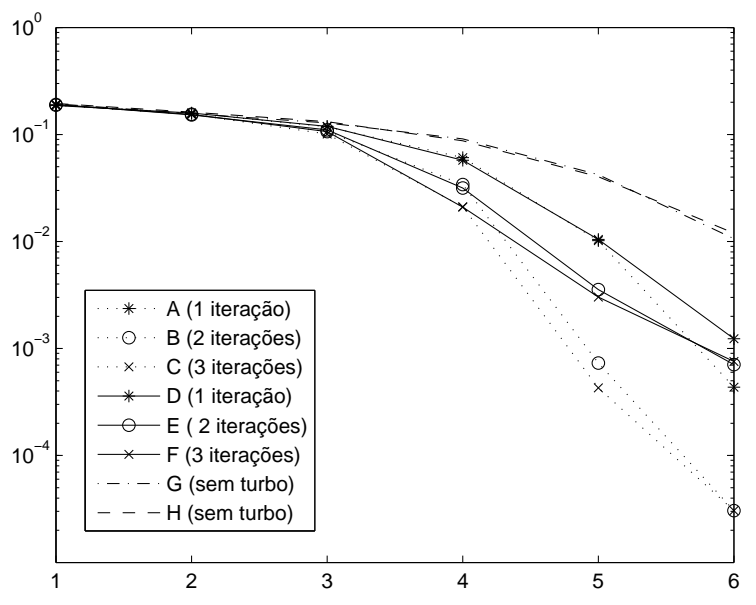

Fig. 10. Curvas relacionadas ao usuário 1. Os casos para os quais $\mathcal{C}_{1}^{-}=\mathcal{C}_{1}^{\mid}=\mathcal{C}_{2}^{-}=\mathcal{C}_{2}^{\mid}$têm matrizes geradoras polinomiais $G(D)=$ $\left[\begin{array}{cc}1 & \frac{1+D+D^{3}}{1+D^{2}+D^{3}}\end{array}\right]$ estão ilustrados em: A, B, C e G; Os casos para os quais $\mathcal{C}_{1}^{-}=\mathcal{C}_{1}^{\mid}=\mathcal{C}_{2}^{-}=\mathcal{C}_{2}^{\mid}$têm matrizes geradoras polinomiais $G(D)=$ $\left[\begin{array}{cc}1 & \frac{1+D+D^{2}+D^{3}}{1+D+D^{3}}\end{array}\right]$ estão ilustrados em: D, E, F e H.

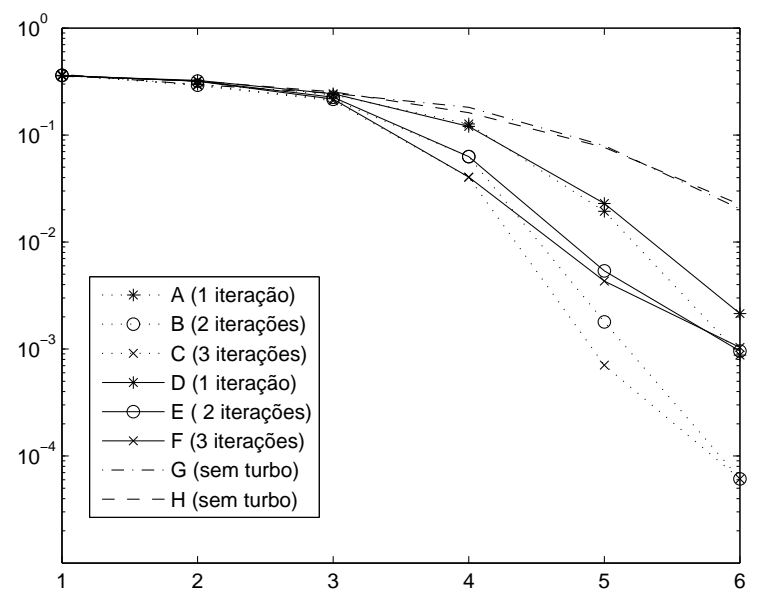

Fig. 11. Curvas relacionadas ao usuário 2. Os casos para os quais $\mathcal{C}_{1}^{-}=\mathcal{C}_{1}^{\mid}=\mathcal{C}_{2}^{-}=\mathcal{C}_{2}^{\mid}$têm matrizes geradoras polinomiais $G(D)=$ $\left[1 \frac{1+D+D^{3}}{1+D^{2}+D^{3}}\right]$ estão ilustrados em: A, B, C e G; Os casos para os quais $\mathcal{C}_{1}^{-}=\mathcal{C}_{1}^{\mid}=\mathcal{C}_{2}^{-}=\mathcal{C}_{2}^{\mid}$têm matrizes geradoras polinomiais $G(D)=$ $\left[\begin{array}{cc}1 & \frac{1+D+D^{2}+D^{3}}{1+D+D^{3}}\end{array}\right]$ estão ilustrados em: D, E, F e H. 
XXV SIMPÓSIO BRASILEIRO DE TELECOMUNICAÇÕES - SBrT’07, 03-06 DE SETEMBRO DE 2007, RECIFE, PE

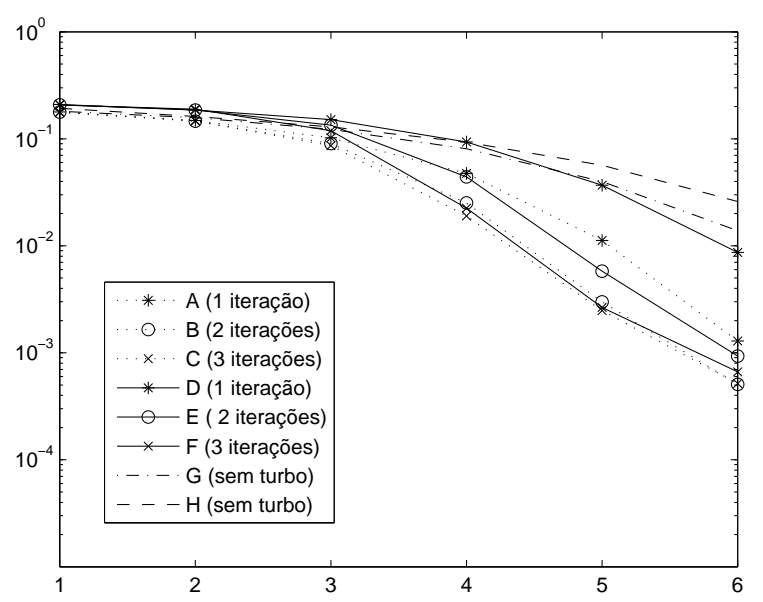

Fig. 12. Curvas relacionadas ao usuário 1. Os casos para os quais $\mathcal{C}_{1}^{-}=\mathcal{C}_{1}^{\mid}=\mathcal{C}_{2}^{-}=\mathcal{C}_{2}^{\mid}$têm matrizes geradoras polinomiais $G(D)=$ $\left[1 \frac{1+D^{2}}{1+D+D^{2}}\right]$ estão ilustrados em: A, B, C e G; Os casos para os quais $\mathcal{C}_{1}^{-}=\mathcal{C}_{1}^{\mid}$têm matrizes geradoras polinomiais $G(D)=\left[\begin{array}{ll}1 & \frac{1+D^{2}}{1+D+D^{2}}\end{array}\right] \mathrm{e}$ $\mathcal{C}_{2}^{-}=\mathcal{C}_{2}^{\mid}$têm matrizes geradoras polinomiais $G(D)=\left[\begin{array}{ll}1 & \frac{D+D^{2}}{1+D+D^{2}}\end{array}\right]$ estão
ilustrados em: D, E, F e H.

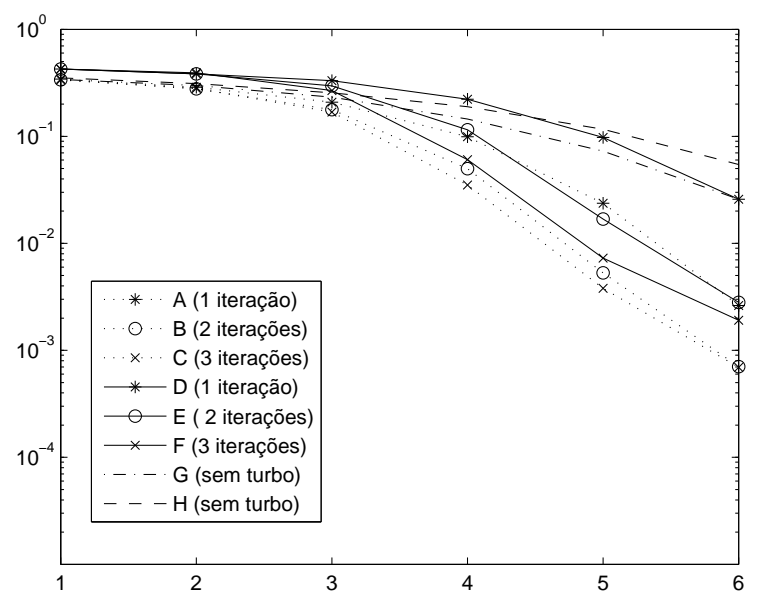

Fig. 13. Curvas relacionadas ao usuário 2. Os casos para os quais $\mathcal{C}_{1}^{-}=\mathcal{C}_{1}^{\mid}=\mathcal{C}_{2}^{-}=\mathcal{C}_{2}^{\mid}$têm matrizes geradoras polinomiais $G(D)=$ $\left[1 \frac{1+D^{2}}{1+D+D^{2}}\right]$ estão ilustrados em: A, B, C e G; Os casos para os quais $\mathcal{C}_{1}^{-}=\mathcal{C}_{1}^{\mid}$têm matrizes geradoras polinomiais $G(D)=\left[\begin{array}{ll}1 & \frac{1+D^{2}}{1+D+D^{2}}\end{array}\right] \mathrm{e}$ $\mathcal{C}_{2}^{-}=\mathcal{C}_{2}^{\mid}$têm matrizes geradoras polinomiais $G(D)=\left[\begin{array}{cc}1 & \frac{D+D^{2}}{1+D+D^{2}}\end{array}\right]$ estão ilustrados em: D, E, F e H. 